\title{
Wir schauen hin!
}

\author{
Zwölf Verbände, Organisationen und Institutionen \\ haben eine Charta zur Prävention von sexueller \\ Ausbeutung, Missbrauch und anderen Grenzver- \\ letzungen gegenüber Menschen mit Behinderung \\ erarbeitet.
}

Präsident der Schweizerischen Arbeitsgemeinschaft von Ärzten für Menschen mit geistiger oder mehrfacher Behinderung SAGB/ ASHM
Korrespondenz:

Dr. med. Felix Brem Facharzt für Psychiatrie und Psychotherapie FMH Ärzte im Zentrum Rathausstrasse 17 $\mathrm{CH}-8570$ Weinfelden felix.brem[at]hin.ch
Am 25.11.2011 haben zwölf Verbände, Organisationen und Institutionen, darunter die SAGB/ ASHM (Schweizerische Arbeitsgemeinschaft von Ärzten für Menschen mit geistiger oder mehrfacher Behinderung) die Charta zur Prävention von sexueller Ausbeutung, Missbrauch und anderen Grenzverletzungen gegenüber Menschen mit Behinderung den Medien vorgestellt. Sie fordern eine NullToleranz-Politik und setzen vor allem bei den Mitarbeitenden und bei der Stärkung der Personen mit besonderem Unterstützungsbedarf an. Ihre wichtigste Botschaft lautet: «Wir schauen hin! Und zwar gemeinsam.»

Auslöser zum Zusammenschluss der Verbände war das Aufdecken der erschreckend hohen Zahl von Missbrauchshandlungen durch den Sozialtherapeuten HS an Menschen mit Behinderung. Die in einer verbandsübergreifenden Arbeitsgruppe erarbeitete Charta umfasst 10 Grundsätze, welche die vier Themenbereiche Präventionskonzepte, Stärkung der Personen mit Unterstützungsbedarf, Schlüsselrolle der Mitarbeitenden und Einrichtung einer internen Meldestelle und externen Ombudsstelle betreffen. Die Grundsätze gelten für alle Personen, die in Institutionen oder Organisationen tätig sind oder betreut werden.
Die Verbände, Organisationen und Institutionen machen unmissverständlich klar, «dass wir keine sexuelle Ausbeutung, keinen Missbrauch und keine anderen Grenzverletzungen dulden, dass wir jedem Verdacht nachgehen und dass wir eine Null-Toleranz-Politik verfolgen», erläuterte Ivo Lötscher, Leiter der Arbeitsgruppe und Geschäftsführer von INSOS Schweiz. Diese Haltung gelte es nun nach innen und nach aussen $\mathrm{zu}$ kommunizieren und $\mathrm{zu}$ leben. «Die auf Unterstützung angewiesenen Personen sollen wissen: Wir schauen hin. Und potentielle Täter müssen wissen: Bei uns gilt Null-Toleranz.»

Wir Ärzte werden unseren Beitrag insbesondere durch erhöhte Achtsamkeit auf sämtliche direkten oder indirekten Hinweise behinderter Menschen auf Misshandlungen oder Missbrauch sowie auf Abgrenzungsprobleme von Mitarbeitenden der Institutionen leisten; darüber hinaus wollen wir uns aber auch generell mehr für die Alltagssituation in Behinderteninstitutionen interessieren. Auch sind wir zu verstärkter und verbesserter Zusammenarbeit aufgerufen. Die SAGB/ASHM wird sich vertieft mit dieser Thematik auseinandersetzen und weiterhin aktiv in der Arbeitsgruppe mitwirken.

Informationen zur Charta finden Sie unter www.charta-praevention.ch

\section{Charta zur Prävention von sexueller Ausbeutung, Missbrauch und anderen Grenzverletzungen gegenüber Menschen mit Behinderung}

Verbandsübergreifende Arbeitsgruppe Prävention
Die unterzeichnenden Verbände, Institutionen und Organisationen bekennen sich zu den folgenden Grundsätzen zur Prävention von sexueller Ausbeutung, Missbrauch und anderen Grenzverletzungen. Die Grundsätze gelten für alle Personen, die in unseren Institutionen und Organisationen tätig sind oder betreut werden.

\section{Präventionskonzept}

1. Jede unserer Institutionen und Organisationen verfügt über Konzepte, Strategien und Mass- nahmenpläne zur Prävention von sexueller Ausbeutung, Missbrauch und anderen Grenzverletzungen. Das Vorgehen bei einem Verdacht oder einem Fall von sexueller Ausbeutung ist geregelt und allen Mitarbeitenden, den betreuten Personen und den Angehörigen bekannt. Jedem Verdacht wird nachgegangen (Null-Toleranz-Politik).

2. Wir tragen mit regelmässiger interner und externer Kommunikation dazu bei, die notwendige Sensibilität hoch zu halten. 


\section{Stärkung der Personen mit besonderem Unterstützungsbedarf}

3. Die Förderung der Selbstkompetenzen der Personen mit besonderem Unterstützungsbedarf in unseren Institutionen und Organisationen nimmt in Bezug auf den Umgang mit Nähe und Distanz, auf das Setzen von Grenzen sowie auf die eigene Sexualität einen hohen Stellenwert ein. Sie müssen wissen, wie sie sich gegen eine Verletzung ihrer persönlichen Integrität zur Wehr setzen können. in unseren Institutionen und Organisationen engagieren.

9. Wir verfassen wahrheitsgetreue, vollständige Zeugnisse und Einsatzbestätigungen und geben ebensolche Referenzauskünfte.

\section{Interne Meldestelle und externe \\ Ombudsstelle}

10. In unseren Institutionen und Organisationen gibt es eine interne, niederschwellige Meldestelle mit einer fachlich kompetenten Ansprech-

\section{Wir schauen hin! Wir dulden keine sexuelle Ausbeutung, keinen Missbrauch und keine anderen Grenzverletzungen.}

4. Personen mit hoher Abhängigkeit von Betreuung und Unterstützung sind in diese Förderung einbezogen und werden ihren Möglichkeiten entsprechend befähigt, Abwehr zum Ausdruck zu bringen und Grenzverletzungen zu signalisieren. Bei dieser besonders gefährdeten Personengruppe ziehen wir das persönliche Umfeld (Angehörige, Bezugspersonen) in die Präventionsarbeit mit ein.

\section{Schlüsselrolle der Mitarbeitenden}

5. Bei der Personalgewinnung und -auswahl ist gründlich und achtsam vorzugehen. Die Einreichung eines Strafregisterauszugs ist Anstellungsvoraussetzung für Mitarbeitende, die in direktem Kontakt mit Personen mit besonderem Unterstützungsbedarf stehen. Die Arbeitgebenden prüfen die Zeugnisse sorgfältig (Vollständigkeit) und holen vor der Anstellung Referenzen ein, welche auch zum Umgang mit Nähe und Distanz Auskunft geben.*

6. Bei der Anstellung unterschreiben die neuen Mitarbeitenden unserer Institutionen und Organisationen eine Selbstverpflichtung. Darin verpflichten sie sich, sich aktiv an der Realisierung der Null-Toleranz-Politik zu beteiligen. Sie anerkennen das Präventionskonzept als Teil des Arbeitsvertrags.

7. In unseren Institutionen und Organisationen wird eine Kultur des aufmerksamen Hinschauens und der Transparenz gepflegt. Wir trennen uns von Mitarbeitenden, welche sich dieser Kultur entziehen oder widersetzen.

8. Wir führen regelmässig Weiterbildungen zum Thema «sexuelle Ausbeutung, Missbrauch und andere Grenzverletzungen» durch und bieten diese auch sämtlichen Freiwilligen an, die sich person, deren Auftrag (als Teil des Präventionskonzeptes) den Mitarbeitenden, den Personen mit besonderem Unterstützungsbedarf sowie den gesetzlichen Vertretungen und Angehörigen bekannt ist. Ebenfalls haben alle Personen die Möglichkeit, sich an eine externe Stelle zu wenden.

Die Charta wurde bisher von folgenden Verbänden, Organisationen und Institutionen ratifiziert:

Agogis, Berufliche Bildung im Sozialbereich

Autismus Schweiz

AvenirSocial, Soziale Arbeit Schweiz

Vereinigung Cerebral Schweiz

Curaviva Schweiz, Verband Heime und Institutionen Schweiz

Heimverband Bern

Insieme Schweiz, Schweiz. Vereinigung der Elternvereine für Menschen mit geistiger Behinderung

Insos Schweiz, Nationaler Branchenverband der Institutionen für Menschen mit Behinderung

Procap Schweiz - Für Menschen mit Handicap

Pro Infirmis - Die Organsiation für behinderte Menschen

SAGB, Schweizerische Arbeitsgemeinschaft von Ärzten für Menschen mit geistiger oder mehrfacher Behinderung

VAHS, Verband für anthroposophische Heilpädagogik und Sozialtherapie in der Schweiz der Verhältnismässigkei bei Kurzeinsätzen und freiwilligen Mitarbeitenden 